\title{
Atitudes e comportamentos dos diabéticos acerca das estratégias de prevenção e controle clínico do diabetes
}

\author{
Attitudes and behaviors of diabetics about the diabetes clinical \\ prevention and control strategies
}

\section{Laís Lima De Oliveira ${ }^{1}$, Gêniton Santos Lima ${ }^{2}$, Tiago Da Silva Nunes ${ }^{3}$, Tauanny Aragão De Moura ${ }^{4}$, Diego Moura Tanajura ${ }^{5}$}

Oliveira LL, Lima GF, Nunes TS, Moura TA, Tanajura DM. Atitudes e comportamentos dos diabéticos acerca das estratégias de prevenção e controle clínico do diabetes / Attitudes and behaviors of diabetics about the diabetes clinical prevention and control strategies. Rev Med (São Paulo). 2019 jan.-fev.;98(1):16-22.

RESUMO: Introdução: O Diabetes Mellitus (DM) constitui um dos principais agravos de saúde pública das doenças crônicas não transmissíveis no mundo. Atitudes adequadas dos diabéticos frente às recomendações dos profissionais da saúde são imprescindíveis para sucesso terapêutico. Ademais, o nível de conhecimento sobre a doença constitui um dos pontos principais para prevenção de complicações do diabetes. Objetivo: Avaliar atitudes e comportamentos dos diabéticos acerca das estratégias de prevenção e suas influências no controle clínico do diabetes. Casuistica e Métodos: Estudo transversal descritivo e exploratório realizado em duas Unidades Básicas de Saúde no interior do estado de Sergipe. A coleta de dados se deu através de questionários e realização do exame clínico. Resultados: Ao total, foram aplicados 82 questionários onde todos possuíam o DM tipo 2. A maioria dos pacientes era do sexo feminino (75,6\%) com média de idade de $61,74 \pm 13,06$ anos, $39 \%$ analfabetos e apenas $15 \%$ trabalham. Foi observado excesso de peso em $66 \%$ e sedentarismo em $74 \%$ dos pacientes. Cerca de $10 \%$ apresentam alguma complicação do diabetes, $8 \%$ afirmaram não fazer tratamento conforme recomendado e 5\% apresentam algum grau de amputação de membros inferiores associado ao DM. Mais de $90 \%$ desconheciam sinais, causas e manejo da hipoglicemia e apenas 18,29\% participaram de alguma atividade educativa sobre o cuidado com o pé diabético. Conclusão: Os resultados remetem à necessidade da realização de estratégias preventivas, objetivando a educação em saúde e promoção, para aumentar o conhecimento, e melhorar atitudes e comportamento dos pacientes diabéticos.

Descritores: Diabetes mellitus; Atitude; Autocuidado; Conhecimento; Educação em saúde.

\begin{abstract}
Introduction: Diabetes Mellitus (DM) is one of the main problem of public health among the chronic non transmissible diseases in several countries. The diabetic's attitudes to the health professionals' recommendations are essential for the success therapeutic. In addition, the knowledge about some disease aspects is one of the main points for the prevention diabetes complications. Objective: Evaluate attitudes and behaviors towards prevention strategies adopted and their influence on the clinical control of diabetes. Patients and Methods: Descriptive and exploratory cross-sectional study developed on two Public Basic Health Units in the interior of the state of Sergipe. The collection of data was done through a questionnaire and the clinical exam. Results: A total of 82 questionnaires were applied and all patients had DM type 2 . The most of them were female $(75.6 \%)$ with mean age of $61,74 \pm 13,06$ years, $39 \%$ are illiterate and only $15 \%$ are economically active. Overweight was observed in $66 \%$ of diabetics and sedentary lifestyle in $74 \%$ of them. About $10 \%$ had some type of diabetes complication, $8 \%$ referred do not follow treatment properly and 5\% already presents some level of lower limb amputation associated with diabetes. More than $90 \%$ not had enough knowledge about the signs, causes and management of hypoglycemia, and only $18.29 \%$ participated of educational activities about diabetic foot care. Conclusion: The results show the necessity to develop preventive strategies focused on health education and promotions to increase knowledge, attitudes and behaviors of diabetic patients.
\end{abstract}

Keywords: Diabetes mellitus; Attitude; Self-care; Knowledge; Health education.

1. Graduada em Enfermagem, Universidade Federal de Sergipe (UFS). Lagarto-Sergipe, Brasil. ORCID: https://orcid.org/0000-0002-3736-5754. Email: lais.lima.oliveira@gmail.com.

2. Agente Comunitário de Saúde, Secretaria Municipal de Saúde, Frei Paulo-Sergipe, Brasil. ORCID: https://orcid.org/0000-0002-6780-7314. Email: geniton.lima@gmail.com.

3. Graduando em Medicina, Universidade Federal de Sergipe (UFS). Aracaju-Sergipe, Brasil. ORCID: https://orcid.org/0000-0002-8698-6960. Email: tnunes008@gmail.com.

4. Médica Especialista em Medicina de Família. Residente em Geriatria pela Universidade de São Paulo (USP). São Paulo-São Paulo, Brasil. ORCID: https://orcid.org/0000-0003-1643-8624. Email: taumoura@hotmail.com.

5. Professor Doutor do Departamento de Educação em Saúde da Universidade Federal de Sergipe (UFS). Lagarto-Sergipe, Brasil. ORCID: https://orcid. org/0000-0001-8135-3745. Email: diegomouratanajura@gmail.com.

Endereço para correspondência: Diego Moura Tanajura. Universidade Federal de Sergipe; Campus Prof. Antônio Garcia Filho. Av. Governador Marcelo Déda, 13, Centro. Lagarto - Sergipe, Brasil. CEP: 49400-000. Email: Diegomouratanajura@gmail.com. 


\section{INTRODUÇÃO}

Diabetes Mellitus (DM) consiste num agrupamento heterogêneo de disfunções metabólicas que apresenta quadros de hiperglicemia resultante de defeito na produção, secreção e/ou ação da insulina. Os tipos de DM predominantes na população mundial incluem o tipo 1 e tipo 2, correspondendo a aproximadamente $5 \%$ e $95 \%$ dos casos, respectivamente ${ }^{1}$. É considerado um problema de saúde pública, com uma prevalência estimada de 415 milhões de adultos com a doença. $\mathrm{O}$ Brasil ocupa o quarto lugar no mundo em número de casos, com 14,3 milhões de pessoas diabéticas ${ }^{2}$. No estado de Sergipe, segundo dados do Vigitel (Vigilância de fatores de risco e proteção para doenças crônicas por inquérito telefônico) do ano de 2010, a capital Aracaju se encontra em $5^{\circ}$ lugar geral no ranking de diagnóstico do DM, em adultos acima de 18 anos de idade ${ }^{3}$.

O conhecimento dos pacientes diabéticos sobre a sua doença influencia diretamente no monitoramento glicêmico domiciliar. Conhecer os fatores de risco do DM, os sinais de hiper ou hipoglicemia, os sinais de lesão em órgãos alvo e o tratamento nutricional adequado é estritamente necessário para o tratamento precoce e prevenção das complicações advindas com o $\mathrm{DM}^{4}$. No entanto, segundo estudo realizado em seis unidades básicas de um município brasileiro, mais de $50 \%$ dos diabéticos demonstraram ter conhecimento e compreensão insuficientes em relação à doença, principalmente sobre as possíveis complicações do diabetes ${ }^{5}$. Além disso, outros estudos têm revelado superficialidade do conhecimento dos diabéticos acerca do tratamento e das mudanças de estilo de vida propostas. Esta situação pode interferir na aplicabilidade e na eficácia das recomendações quanto à prevenção de complicações ${ }^{6,7}$.

A alta morbimortalidade do DM é consequência das complicações crônicas advindas com o avançar da doença. As incidência e prevalência de eventos macrovasculares, como a doença arterial coronária, doença arterial periférica e acidentes cerebrovasculares, chegam a dobrar em se tratando de pacientes diabéticos comparado aos não diabéticos ${ }^{8}$. Já a retinopatia diabética apresenta prevalência entre 7 a 39\% de casos em pacientes diabéticos e corresponde a uma das principais causas evitáveis de cegueira9 ${ }^{9}$, enquanto que a nefropatia diabética é uma das principais causas de base para a doença renal crônica no Brasil ${ }^{10}$. Além disso, merece destaque a neuropatia diabética pelo seu potencial incapacitante, pois dados demonstraram que mais de 50\% dos pacientes submetidos à amputação de membros possuíam algum grau de neuropatia associada ao diabetes ${ }^{11}$.

Diante do exposto, o objetivo do estudo foi verificar as atitudes dos diabéticos frente às estratégias de promoção à saúde e prevenção de complicações associadas ao diabetes propostas em duas Unidades de Saúde da Família do interior sergipano. Com isso, é esperado oferecer subsídios necessários para o planejamento de novas ações com vistas ao controle adequado do diabetes.

\section{CASUÍSTICA E MÉTODOS}

Trata-se de um estudo transversal de caráter descritivo e exploratório, o qual avalia as atitudes dos diabéticos frente às estratégias de promoção à saúde e prevenção de complicações associadas ao diabetes adotadas nos municípios de Frei Paulo e Lagarto, estado de Sergipe. As cidades de Frei Paulo, situada a $64 \mathrm{~km}$ de distância da capital Aracaju, e Lagarto, situada a $65 \mathrm{~km}$ da capital, contam com uma população estimada pelo Instituto Brasileiro de Geografia e Estatística (IBGE) de 15.200 e 103.188 habitantes no ano de 2016, respectivamente.

A coleta de dados foi realizada em duas Unidades Básicas de Saúde das respectivas cidades. A primeira, Clínica de Saúde da Família Edelmira Barbosa Dantas de Oliveira, na cidade de Frei Paulo-SE, que presta atendimento a toda a população diabética da zona urbana da cidade. A segunda, a Unidade Básica de Saúde Maria do Carmo Alves, a qual concentra a segunda maior população de cobertura na zona urbana de Lagarto-SE.

A amostragem utilizada foi por conveniência e os dados foram coletados antes ou depois da consulta de rotina do programa Hiperdia. O questionário aplicado foi estruturado com questões referentes às características sociodemográficas incluindo sexo, idade, estado civil, escolaridade, renda e trabalho (exercício de algum tipo de atividade remunerada na época da entrevista - aposentados, donas de casa e pensionistas foram classificadas como pessoas que não trabalham). As variáveis utilizadas para avaliar as características dos pacientes com DM foram classificação do DM, local de acompanhamento clínico, profissional de referência, tempo de diagnóstico, tipo de tratamento, segue o tratamento de forma adequada, uso do glicosímetro, prática de atividade física, complicações e internação. Para a avaliação do conhecimento do diabético sobre sua doença foi avaliado o conhecimento da definição de hiper e hipoglicemia, participação em atividades educativas, conhecimento sobre o autoexame e a prática do mesmo.

Os critérios de inclusão foram: ter diagnóstico de DM1 ou DM2 e consentirem em participar do estudo através da assinatura do TCLE (Termo de Consentimento Livre e Esclarecido). Foram excluídos os pacientes incapacitados de fornecer as informações solicitadas na coleta de dados, como idade avançada que, associada a problemas de desordem cognitiva, os impossibilitavam de responder as perguntas propostas pelo formulário.

Após a realização da entrevista, os pacientes foram submetidos a um exame clínico avaliando-se: peso em $\mathrm{Kg}$, mensurado com roupas leves, sem calçados e os pés justapostos, em balança com precisão de $100 \mathrm{~g}$ e altura em centímetros $(\mathrm{cm})$, medida em estadiômetro de 
parede, com precisão de $1 \mathrm{~mm}$. Para avaliação do estado nutricional utilizou-se o índice de massa corporal (IMC) definido pela formula peso/(altura) ${ }^{2}$ e a estratificação do IMC de sobrepeso aos diferentes graus de obesidade foi realizada com base na classificação proposta pela Associação Brasileira para o Estudo da Obesidade e da Síndrome Metabólica ${ }^{12}$. Os participantes também foram submetidos ao exame clínico do pé diabético o qual avaliou a presença de deformidades (principalmente a Artropatia de Charcot), presença de ulcerações ou fatores de risco para o desenvolvimento destas, tais como: pele seca, presença de micoses ou fissuras.

Os dados foram digitados em planilhas do Microsoft Office Excel 2007. A análise dos dados foi descritiva com medida de frequência relativa e absoluta. O estudo atende as normas do Conselho Nacional de Saúde e foi aprovado pelo Comitê de Ética e Pesquisa do Hospital Universitário da Universidade Federal de Sergipe (CAAE: 48371115100005546).

\section{RESULTADOS}

A amostra inicial foi composta de 88 diabéticos, da qual foram excluídos seis devido ao preenchimento incompleto dos dados. Do total de 82 participantes, $75 \%$ eram mulheres, a média de idade foi de $61,74 \pm 13,06$ anos (média \pm desvio padrão). Aproximadamente 39\% dos entrevistados eram analfabetos e outros 39\% não completaram o primeiro grau. Além disso, apenas 15\% desenvolvem algum tipo de atividade remunerada, sendo a renda média composta de até dois salários mínimos em aproximadamente $93 \%$ da amostra (Tabela 1 ).

Tabela 1. Características sociodemográficas dos pacientes diabéticos investigados em Lagarto e Frei Paulo - SE, 2016

\begin{tabular}{l|c|c}
\hline Variáveis & $\mathbf{n}$ & $\mathbf{\%}$ \\
\hline Sexo & 62 & 75,61 \\
Feminino & 20 & 24,39 \\
Masculino & 39 & 47,56 \\
\hline Estado civil & 11 & 13,41 \\
Casado/União estável & 25 & 30,49 \\
Solteiro & 07 & 8,54 \\
Viúvo & 32 & 39,02 \\
Divorciado & 32 & 39,02 \\
\hline Escolaridade & 13 & 15,85 \\
Analfabeto & 01 & 1,22 \\
Primeiro grau incompleto & 03 & 3,66 \\
Primeiro grau completo & 01 & 1,22 \\
Segundo grau incompleto & & \\
Segundo grau completo & 13 & 15,85 \\
Superior completo & 69 & 84,15 \\
\hline Trabalho & & 9,32 \\
Sim & 76 & \\
Não & 06 & \\
\hline Renda & & \\
Até 2 salários mínimos & & \\
Acima de 2 salários & & \\
mínimos & & \\
\hline
\end{tabular}

Todos os participantes referiram diagnóstico de DM tipo 2 e $81,71 \%$ destes sãos acompanhados exclusivamente pelo SUS e $86,59 \%$ por clínicos gerais. O tipo de tratamento predominante foi composto pelo uso exclusivo de hipoglicemiantes orais em $89,02 \%$ da amostra e $9,76 \%$ já fazem uso da aplicação da insulina concomitante à medicação oral (Tabela 2).

Tabela 2. Acompanhamento clínico dos pacientes diabéticos investigados em Lagarto e Frei Paulo - SE, 2016

\begin{tabular}{l|c|c}
\hline Variáveis & $\mathbf{n}$ & $\mathbf{\%}$ \\
\hline Classificação do diabetes & & \\
$\quad$ Diabetes tipo 1 & 0 & 0 \\
$\quad$ Diabetes tipo 2 & 82 & 100 \\
\hline Acompanhamento & 67 & 81,71 \\
SUS & 7 & 8,54 \\
Privado & 8 & 9,75 \\
SUS/Privado & & \\
& & \\
\hline Profissional de referência & 71 & 86,59 \\
Clínico geral & 11 & 13,41 \\
Endocrinologista & & \\
& & \\
\hline Tempo de diagnóstico (anos) & 9 & 10,97 \\
$\quad<1$ & 29 & 35,36 \\
1 a 5 & 28 & 34,15 \\
6 a 10 & 16 & 19,51 \\
>10 & & \\
\hline Tipo de tratamento & 73 & 89,02 \\
Hipoglicemiantes & 8 & 9,76 \\
Hipoglicemiantes/Insulina & 1 & \\
Não farmacológico & & \\
\hline
\end{tabular}

Noventa porcento dos participantes relataram não apresentar complicações advindas do DM e cerca de 93\% afirmaram seguir o tratamento conforme recomendação médica. No entanto, $73 \%$ não fazem uso do glicosímetro regularmente e $6 \%$ tiveram episódios de internação hospitalar por descompensação aguda do DM nos últimos doze meses que antecederam a entrevista. Ademais, merece destaque o excesso de peso observado em cerca de $66 \%$ dos participantes. Além disso, $74 \%$ não praticam atividade física (Tabela 3).

Referente à avaliação clínica dos pés, foi possível observar em $45 \%$ da amostra a presença de fatores predisponentes de complicações do pé diabético, tais como: pele seca, micoses, fissuras, entre outros. Cerca de $12 \%$ apresentaram histórico anterior de ulcerações e $8 \%$ apresentaram deformidades ósseas nos pés. Aproximadamente 5\% dos participantes já evoluíram para graus variados de amputação dos membros inferiores (Tabela 4). 
Oliveira LL, et al. Atitudes e comportamentos dos diabéticos acerca das estratégias de prevenção e controle.

Tabela 3. Comportamentos e atitudes dos diabéticos investigados acerca da terapia adotada. Lagarto e Frei Paulo - SE, 2016

\begin{tabular}{|c|c|c|}
\hline Variáveis & $\mathbf{n}$ & $\%$ \\
\hline $\begin{array}{l}\text { Seguimento do tratamento } \\
\text { Recomendado } \\
\text { Não recomendado }\end{array}$ & $\begin{array}{c}76 \\
6\end{array}$ & $\begin{array}{r}92,68 \\
7,32\end{array}$ \\
\hline $\begin{array}{l}\text { Uso do glicosímetro } \\
\text { Sim } \\
\text { Não }\end{array}$ & $\begin{array}{l}22 \\
60\end{array}$ & $\begin{array}{l}26,83 \\
73,17\end{array}$ \\
\hline $\begin{array}{l}\text { Classificação IMC } \\
\text { Ideal } \\
\text { Sobrepeso } \\
\text { Obesidade grau I } \\
\text { Obesidade grau II } \\
\text { Obesidade grau III }\end{array}$ & $\begin{array}{l}28 \\
22 \\
16 \\
08 \\
08\end{array}$ & $\begin{array}{c}34,14 \\
26,83 \\
19,51 \\
9,76 \\
9,76\end{array}$ \\
\hline $\begin{array}{l}\text { Atividade Física } \\
\text { Sim } \\
\text { Não }\end{array}$ & $\begin{array}{l}21 \\
61\end{array}$ & $\begin{array}{l}25,61 \\
74,39\end{array}$ \\
\hline $\begin{array}{l}\text { Presença de complicações } \\
\text { Sim } \\
\text { Não }\end{array}$ & $\begin{array}{c}8 \\
74\end{array}$ & $\begin{array}{c}9,76 \\
90,24\end{array}$ \\
\hline $\begin{array}{l}\text { Internação hospitalar } \\
\text { (últimos } 12 \text { meses) }\end{array}$ & & \\
\hline $\begin{array}{l}\text { Sim, por descompensação } \\
\text { diabética }\end{array}$ & 5 & 6,10 \\
\hline Sim, por outras causas & 6 & 7,32 \\
\hline Não & 71 & 86,58 \\
\hline
\end{tabular}

Tabela 4. Avaliação do pé diabético em portadores de DM investigados. Lagarto e Frei Paulo - SE, 2016

\begin{tabular}{l|c|c}
\hline Variáveis & $\mathbf{n}$ & $\mathbf{\%}$ \\
\hline Complicações no pé & 37 & 45,12 \\
Sim & 45 & 54,88 \\
Não & 10 & 12,20 \\
\hline Histórico de ulcerações & 72 & 87,80 \\
Sim & 5 & 6,10 \\
Não & 2 & 2,44 \\
\hline Apresenta deformidades & 75 & 91,46 \\
Sim, em um membro & & \\
Sim, nos dois membros & 4 & 4,88 \\
Não & 78 & 95,12 \\
\hline Amputações &
\end{tabular}

Quanto à avaliação do conhecimento dos diabéticos investigados no estudo, acerca da doença, 97,56\% e $93,9 \%$ não souberam definir de forma correta os sinais e causas da hipoglicemia e hiperglicemia, respectivamente. Apenas 18,29\% afirmaram já ter participado de algum tipo de atividade educativa a respeito do cuidado com o pé diabético e somente $9 \%$ conhecem e realizam o autoexame dos pés (Tabela 5).
Tabela 5. Conhecimento dos diabéticos investigados sobre a sua doença. Lagarto e Frei Paulo - SE, 2016

\begin{tabular}{|c|c|c|}
\hline Variáveis & $\mathbf{n}$ & $\%$ \\
\hline $\begin{array}{l}\text { Define Hipoglicemia } \\
\text { Sim } \\
\text { Não }\end{array}$ & $\begin{array}{c}2 \\
80 \\
\end{array}$ & $\begin{array}{c}2,44 \\
97,56\end{array}$ \\
\hline $\begin{array}{l}\text { Define Hiperglicemia } \\
\text { Sim } \\
\text { Não }\end{array}$ & $\begin{array}{c}5 \\
77\end{array}$ & $\begin{array}{c}6,10 \\
93,90\end{array}$ \\
\hline $\begin{array}{l}\text { Participação em atividade educativa } \\
\text { sobre saúde dos pés } \\
\text { Sim } \\
\text { Não }\end{array}$ & $\begin{array}{l}15 \\
67\end{array}$ & $\begin{array}{l}18,29 \\
81,71\end{array}$ \\
\hline $\begin{array}{l}\text { Conhece o autoexame do pé } \\
\text { Sim } \\
\text { Não }\end{array}$ & $\begin{array}{c}8 \\
74\end{array}$ & $\begin{array}{c}9,76 \\
90,24\end{array}$ \\
\hline $\begin{array}{l}\text { Realiza o autoexame do pé } \\
\text { Sim } \\
\text { Não }\end{array}$ & $\begin{array}{c}8 \\
74\end{array}$ & $\begin{array}{c}9,76 \\
90,24\end{array}$ \\
\hline
\end{tabular}

\section{DISCUSSÃO}

Com o avanço da prevalência das doenças crônicas não transmissíveis, há de se esperar mudanças de atitude tanto dos pacientes quanto dos profissionais de saúde, perante ao manejo mais adequado destas doenças. Entretanto, o sistema de saúde ainda encontra-se com o foco voltado principalmente para as condições agudas, resultado de longos anos de atuação pelo modelo biomédico de atenção à saúde ${ }^{13}$. O controle clínico das doenças crônicas passa pela necessidade do autocuidado e mudança no estilo de vida do paciente. Assim também acontece com o DM, considerada uma das maiores epidemias mundiais do século $\mathrm{XXI}^{2}$.

No presente estudo, todos os pacientes possuíam o DM do tipo 2, forma clínica mais prevalente na população mundial $^{2,14}$. O predomínio do sexo feminino, também relatado por Oliveira et al. ${ }^{15}$ e Rossaneis et al. ${ }^{16}$, remete a uma maior procura do gênero pelo serviço público de saúde. A média de idade acima de 60 anos confirma o caráter progressivo da doença ${ }^{17}$. Além disso, a baixa escolaridade dos diabéticos encontrada na amostra em estudo é citada por outros autores como um fator limitante e que interfere no empoderamento do paciente acerca da própria doença. Embora não seja um fator determinante no aparecimento do diabetes, a escolaridade acaba por interferir no grau de controle clínico da doença ${ }^{13,18}$.

Idade avançada, estado nutricional inadequado, o maior tempo de diagnóstico da doença e a necessidade do uso de insulina associada à terapia oral foram reportados como principais causas no aumento da prevalência de complicações em pacientes diabéticos ${ }^{19}$. Outros estudos apresentaram taxas de prevalência de complicações de três a seis vezes maiores do que a do presente estudo ${ }^{20,21}$. A baixa prevalência de complicações observadas no presente trabalho $(9,76 \%)$ pode ser justificada pelo desconhecimento da população quanto as complicações associadas ao 
diabetes. Por exemplo, a neuropatia diabética, uma das complicações mais prevalentes, se inicia de maneira insidiosa de forma que o paciente só passa a se queixar em estados mais avançados ${ }^{17}$. Dessa forma, a melhor instrução dos pacientes quanto as complicações do diabetes fariam com que estes dessem mais valor as queixas de forma mais precoce e, consequentemente, possibilitaria uma intervenção em uma fase ainda inicial. Isto, por sua vez, pode ser obtido com a realização de campanhas educativas para a população diabética focando num maior esclarecimento sobre a doença e suas complicações.

A análise antropométrica deve fazer parte da rotina de avaliação dos diabéticos já que estudos têm demonstrado associação positiva entre o controle do IMC com os valores de glicose sanguínea ${ }^{22}$. O fato de mais da metade da população se encontrar com sobrepeso ou variados níveis de obesidade pode ser reflexo de diversas lacunas do acompanhamento nutricional desses diabéticos ${ }^{12}$. Dados semelhantes foram encontrados em outro estudo realizado com DM2 da cidade de Aracaju-SE ${ }^{23}$. Além disso, alguns estudos têm revelado que grande parte da população com DM2 é sedentária e apresenta pouca aptidão para prática de atividade física ${ }^{24}$. Situação confirmada também neste estudo onde a grande maioria dos pacientes refere não realizar nenhum tipo de atividade física.

Embora os valores de glicemia sanguínea apresentem relação positiva com a ocorrência de complicações do DM2, bem como seja utilizada para decisão terapêutica no esquema de hipoglicemiantes do paciente, a dosagem de glicemia capilar através da automonitorização destes pacientes ainda é motivo de bastante discussão no meio científico. Nos que fazem uso somente de hipoglicemiantes orais, inexistem evidências que associem a automonitorização com o melhor controle glicêmico, ao contrário do que se observa nos pacientes insulinizados, cuja ausência da automonitorização é critério de exclusão da insulinoterapia ${ }^{17,25}$. O que se observa no presente estudo, porém, é que embora somente $9,76 \%$ dos pacientes estejam em insulinoterapia, $26,83 \%$ realizam a automonitorização da glicemia capilar pelo uso do glicosímetro. Essa quantidade de pacientes que realizam a automonitorização sem indicação terapêutica pode ser justificava pela falta de conhecimento da população sobre a real necessidade dessa monitorização.
A avaliação regular da saúde do pé diabético, embora seja uma prática subutilizada pelos profissionais da saúde, é primordial para a identificação de pacientes com risco de ulcerações, deformidades, calosidades e comprometimento circulatório ${ }^{26}$. Os resultados insatisfatórios encontrados acerca da avaliação clínica do pé diabético remetem uma preocupação importante para saúde pública já que a presença de complicações nos pés podem levar a vários níveis de amputação dos membros inferiores com consequente diminuição da qualidade de vida dos diabéticos e aumento dos gastos públicos ${ }^{27}$.

Contudo, é importante destacar que o autocuidado com os pés é essencial na prevenção de complicações do pé diabético. Já no momento do diagnóstico o paciente deve ser orientado pelo profissional de saúde acerca da importância do autoexame regular dos pés. O baixo grau de conhecimento acerca do cuidado com os pés encontrado nos diabéticos em questão, também relatado por Boell et al. ${ }^{23}$, pode estar associado a diversos fatores tais como: a negligência por parte dos profissionais da saúde no repasse de informações acerca do cuidado adequado ou mesmo a baixa adesão do próprio diabético às recomendações ${ }^{(28)}$.

\section{CONCLUSÃO}

Este estudo possibilitou uma visão geral a respeito de vários aspectos acerca das atitudes dos diabéticos quanto às estratégias de controle clínico adotadas, além de descrever os principais comportamentos de risco encontrados na população que podem levar ao insuficiente controle glicêmico e no aparecimento de complicações advindas com o diabetes. Os dados apresentados fornecem subsídios importantes no desenvolvimento de novas estratégias de acompanhamento dos casos de diabetes nas respectivas áreas de estudo. Além disso, é destacada a importância da educação em saúde como perspectiva de melhorias no controle dos casos de diabetes. Como limitações do estudo vale ressaltar a falta de registros documentais nas unidades estudadas acerca da cobertura populacional regular dos pacientes atendidos pelo programa HiperDia. Outro fator que influenciou diretamente no número amostral se refere à inconstância na realização das consultas do HiperDia.

Contribuições dos autores: Laís Lima de Oliveira: coleta, tabulação, delineamento do estudo, discussão dos achados e redação do manuscrito; Gêniton Santos Lima: coleta e tabulação; Tiago da Silva Nunes: discussão dos achados e redação do manuscrito; Tauanny Aragão de Moura: discussão dos achados e elaboração do manuscrito; Diego Moura Tanajura: orientação do projeto, delineamento do estudo, discussão dos achados e elaboração do manuscrito.

\section{REFERÊNCIAS}

1. Skyler JS, Bakris GL, Bonifacio E, Darsow T, Eckel RH, Groop L, Groop PH, Handelsman Y, Insel RA, Mathieu C, McElvaine AT. Differentiation of diabetes by pathophysiology, natural history, and prognosis. Diabetes. 20171;66(2):24155. doi: https://dx.doi.org/10.2337\%2Fdb16-0806.

2. International Diabetes Federation. IDF diabetes atlas. 7th 
Oliveira LL, et al. Atitudes e comportamentos dos diabéticos acerca das estratégias de prevenção e controle.

ed. Brussels, Belgium; 2015. Available from: https://www. idf.org/e-library/epidemiology-research/diabetes-atlas/13diabetes-atlas-seventh-edition.html.

3. Brasil. Ministério da Saúde. Secretaria de Vigilância em Saúde. Secretaria de Gestão Estratégica e Participativa. VIGITEL Brasil 2010. Vigilância de fatores de risco e proteção para doenças crônicas por inquérito telefônico: estimativas sobre frequência e distribuição sociodemográfica de fatores de risco e proteção para doenças crônicas nas capitais dos 26 Estados brasileiros e no Distrito Federal em 2013. Brasília, DF; 2011. Disponível em: http://bvsms.saude. gov.br/bvs/publicacoes/vigitel_2010.pdf.

4. Chagas IA, Camilo J, Dos Santos MA, Rodgues FF, Arrelias CC, Souza Teixeira CR, Otero LM, Zanetti ML. Conhecimento de pacientes com diabetes sobre tratamento após cinco anos do término de um programa educativo. Rev Esc Enferm USP. 2013;47(5):1137-42. doi: https://doi. org/10.1590/reeusp.v47i5.78072.

5. Oliveira Santos BM, Caixeta AC, Silva AA, de Souza Teixeira CR. Conhecimento e atitudes em diabetes mellitus tipo 2: subsídios para autocuidado e promoção de saúde. Arq Cien Saúde. 2016;23(4):31-6. doi: https://doi. org/10.17696/2318-3691.23.4.2016.443.

6. Oliveira PS, Bezerra EP, Andrade LL, Soares MJ, Costa MM. Contributing factors to the emergence of complications from diabetes mellitus. Rev Enferm UFPE. 2013:5265-73. doi: https://www.doi.org/10.5205/reuol.3452-28790-4ED.0708201326.

7. Andrade Dias JA, Alves Rodrigues R, Nogueira Sales Z, Meira Oliveira Z, Gonçalves Nery PI. Diabetes melitus clients' conceptions about the treatment. J Nursing UFPE. 2016;10(7). doi: https://www.doi.org/ 10.5205/reuol.910680230-1-SM1007201622.

8. Korytkowski MT, Forman DE. Management of atherosclerotic cardiovascular disease risk factors in the older adult patient with diabetes. Diabetes Care. 2017;40(4):476-84. doi: https:// doi.org/10.2337/dc16-0815.

9. Schellini SA, Carvalho GM, Rendeiro FS, Padovani CR, Hirai FE. Prevalence of diabetes and diabetic retinopathy in a Brazilian population. Ophthalmic Epidemiol. 2014;21(1):338. doi: https://doi.org/10.3109/09286586.2013.868004.

10. Siviero PC, Machado CJ, Cherchiglia ML. Insuficiência renal crônica no Brasil segundo enfoque de causas múltiplas de morte. Cad Saude Coletiva. 2014;22(1):75-85. doi: http:// dx.doi.org/10.1590/1414-462X201400010012.

11. Ramos Vieira Santos IC, Freese de Carvalho E, Vieira de Souza W, Cavalcanti de Albuquerque E. Fatores associados a amputações por pé diabético. J Vasc Bras. 2015;14(1). doi: http://dx.doi.org/10.1590/1677-5449.20140049.

12. Associação Brasileira para o Estudo da Obesidade e da Síndrome Metabólica - ABESO. Diretrizes brasileiras de obesidade 2016. 4a ed. São Paulo; 2016. Disponível em: http:// www.abeso.org.br/uploads/downloads/92/57fccc $403 \mathrm{e} 5 \mathrm{da}$. pdf.

13. Taddeo PD, Gomes KW, Caprara A, Gomes AM, Oliveira GC,
Moreira TM. Acesso, prática educativa e empoderamento de pacientes com doenças crônicas. Cien Saude Coletiva. 2012;17:2923-30. Disponível em: https://www.scielosp.org/ pdf/csc/2012.v17n11/2923-2930/pt.

14. American Diabetes Association. Standards of medical care in diabetes - 2014. Diabetes Care. 2014;37(Suppl 1):S14-80. doi: https://doi.org/10.2337/dc14-S014.

15. Rossaneis MA, Haddad MD, Mathias TA, Marcon SS. Differences in foot self-care and lifestyle between men and women with diabetes mellitus. Rev Latino-Am Enfermagem. 2016;24:e2461. doi: http://dx.doi.org/10.1590/15188345.1203.2761.

16. de Oliveira LM, de Souza MF, de Souza LA, da Cruz Melo IR. Adesão ao tratamento dietético e evolução nutricional e clínica de pacientes com diabetes mellitus tipo 2. HU Rev. 2016;42(4):277-82. Disponível em: http://ojs2.ufjf. emnuvens.com.br/hurevista/article/view/2488.

17. Milech A, Angelucci AP, Golbert A, Matheus A, Carrilho AJ, Ramalho AC. Diretrizes da Sociedade Brasileira de Diabetes (2015-2016). São Paulo: AC Farmacêutica; 2016. Disponível em: https://www.diabetes.org.br/profissionais/images/docs/ DIRETRIZES-SBD-2015-2016.pdf.

18. Rodrigues FF, Santos MA, Teixeira CR, Gonela JT, Zanetti ML. Relação entre conhecimento, atitude, escolaridade e tempo de doença em indivíduos com diabetes mellitus. Acta Paul Enfermagem. 2012;25(2):284-90. Disponível em: http:// www.scielo.br/pdf/ape/v25n2/a20v25n2.

19. Santos AD, Cecílio HP, Teston EF, Arruda GO, Peternella FM, Marcon SS. Microvascular complications in type 2 diabetes and associated factors: a telephone survey of selfreported morbidity. Cien Saude Coletiva. 2015;20:761-70. doi: https://doi.org/10.1590/1413-81232015203.12182014.

20. Tracey ML, McHugh SM, Fitzgerald AP, Buckley CM, Canavan RJ, Kearney PM. Risk factors for macro-and microvascular complications among older adults with diagnosed type 2 diabetes: findings from the Irish longitudinal study on ageing. J Diabetes Res. 2016;2016:5975903. doi: http://dx.doi.org/10.1155/2016/5975903.

21. Litwak L, Goh SY, Hussein Z, Malek R, Prusty V, Khamseh ME. Prevalence of diabetes complications in people with type 2 diabetes mellitus and its association with baseline characteristics in the multinational A 1 chieve study. Diabetol Metab Syndr. 2013;5(1):57. doi: https://doi. org/10.1186/1758-5996-5-57.

22. de Jesus DM, Aguiar BG, Dutra CM, Pitombeira PD. Diabetes Mellitus tipo 2: ações de enfermagem na prevenção e controle dos pacientes obesos e com alto índice glicêmico. Rev Acreditação: ACRED. 2016;6(11):20-33. Disponível em: https://dialnet.unirioja.es/servlet/articulo? codigo $=5602107$.

23. de Souza MF, Araújo VF. Adequação do consumo e evolução antropométrica após educação nutricional de pacientes com diabetes mellitus tipo 2. DEMETRA: Alimentação Nutrição Saúde. 2015;10(1):159-72. doi: http://dx.doi.org/10.12957/ demetra.2015.13376.

24. Santos APA. Associação entre atividade física, aptidão 
física e qualidade de vida em pessoas idosas com diabetes Mellitus Tipo 2 [Dissertação]. Évora, Portugal: Universidade de Évora, Escola de Ciências e Tecnologia, Departamento de Desporto e Saúde; 2015. Disponível em: https://dspace. uevora.pt/rdpc/bitstream/10174/14134/1/Dissertação Ana Santos.pdf.

25. Brasil. Portaria GM. 2.583, de 10 de outubro de 2007. Define elenco de medicamentos e insumos disponibilizados pelo Sistema Único de Saúde, nos termos da Lei no ${ }^{\circ} 11.347$, de 2006, aos usuários portadores de diabetes mellitus. Disponível em: http://bvsms.saude.gov.br/bvs/saudelegis/ gm/2007/prt2583_10_10_2007.html.

26. Boell JE, Ribeiro RM, da Silva DM. Fatores de risco para o desencadeamento do pé diabético. Rev Eletrônica Enfermagem. 2014;16(2):386-93. doi: https://doi. org/10.5216/ree.v16i2.20460.

27. Oliveira MA, Francisco PM, Costa KS, Barros MB. Automedicação em idosos residentes em Campinas, São Paulo, Brasil: prevalência e fatores associados. Cad Saude Pública. 2012;28:335-45. Disponível em: https://www.scielosp.org/ scielo.php?pid=S0102-311X2012000200012\&script $=$ sci_ arttext\&tlng=en.

28. Neta R, Sá D, Silva AR, Silva GR. Adesão das pessoas com diabetes mellitus ao autocuidado com os pés. Rev Bras Enferm. 2015;68(1):111-6. doi: http://dx.doi. org/10.1590/0034-7167.2015680115p.

Recebido: 07.08.19

Aceito: 18.03 .19 